\title{
Existence of positive solutions for singular fourth-order three-point boundary value problems
}

Yan Sun ${ }^{1,2^{*}}$ and Cun Zhu ${ }^{2,3}$

\section{"Correspondence:}

sunyan@fudan.edu.cn;

ysun881@sina.com.cn;

ysun@shnu.edu.cn

1 School of Mathematical Sciences,

Fudan University, Shanghai, 200433

P.R. China

${ }^{2}$ Department of Mathematics,

Shanghai Normal University,

Shanghai, 200234, P.R. China

Full list of author information is

available at the end of the article

\begin{abstract}
In this article, we consider the boundary value problem $u^{(4)}(t)+f(t, u(t))=0,0<t<1$, subject to the boundary conditions $u(0)=u^{\prime}(0)=u^{\prime \prime}(0)=0$ and $u^{\prime \prime}(1)-\alpha u^{\prime \prime}(\eta)=\lambda$. In this setting, $0<\eta<1$ and $\alpha \in\left[0, \frac{1}{\eta}\right)$ are constants and $\lambda \in[0,+\infty)$ is a parameter. By imposing a sufficient structure on the nonlinearity $f(t, u)$, we deduce the existence of at least one positive solution to the problem. The novelty in our setting lies in the fact that $f(t, u)$ may be singular at $t=0$ and $t=1$. Our results here are achieved by making use of the Krasnosel'skii fixed point theorem. We conclude with examples illustrating our results and the improvements that they present.
\end{abstract}

MSC: 34B15; 34B25; 34B18

Keywords: cone; positive solution; existence; boundary value problems

\section{Introduction}

In this paper, we consider the following nonlinear singular fourth-order three-point boundary value problem:

$$
\left\{\begin{array}{l}
u^{(4)}(t)+f(t, u(t))=0, \quad 0<t<1, \\
u(0)=u^{\prime}(0)=u^{\prime \prime}(0)=0, \\
u^{\prime \prime}(1)-\alpha u^{\prime \prime}(\eta)=\lambda,
\end{array}\right.
$$

where $0<\eta<1, \alpha \in\left[0, \frac{1}{\eta}\right)$ are constants, $\lambda \in[0,+\infty)$ is a parameter, $f(t, u(t))$ may be singular at $t=0$ and/or $t=1$. Here, by a positive solution we mean a function $u^{*}(t)$ which is positive on $(0,1)$ and satisfies problem (1.1).

The theory of boundary value problems for ordinary differential equations arises in different areas of applied mathematics, physics and so on. The existence of positive solutions for boundary value problems has become an important area of investigation and received a great deal of attention in recent years (see [1-20] and the references cited therein). In [14], by making use of the fixed point theorem and degree theory, Bai and Wang proved the existence, uniqueness and multiplicity of positive solutions for the following fourth-order two-point boundary value problem:

$$
\left\{\begin{array}{l}
u^{(4)}(t)-\lambda f(t, u(t))=0, \quad 0<t<1, \\
u(0)=u(1)=u^{\prime \prime}(0)=u^{\prime \prime}(1)=0 .
\end{array}\right.
$$

(C) 2013 Sun and Zhu; licensee Springer. This is an Open Access article distributed under the terms of the Creative Commons Attribution License (http://creativecommons.org/licenses/by/2.0), which permits unrestricted use, distribution, and reproduction in any medium, provided the original work is properly cited. 
In [8], Yao studied the following nonlinear fourth-order ordinary differential equation:

$$
\left\{\begin{array}{l}
u^{(4)}(t)=f\left(t, u(t), u^{\prime}(t)\right), \quad t \in[0,1] \backslash E, \\
u(0)=u^{\prime}(0)=u^{\prime \prime}(1)=u^{\prime \prime \prime}(1)=0,
\end{array}\right.
$$

where $E \subset[0,1]$ is a closed set with measure zero and the nonlinear term $f(t, x, y)$ may be singular for $t \in E$. The author showed the existence of $n$ positive solutions by constructing a suitable integral equation and applying fixed point theorems on a cone.

In [9], Sun considered the following third-order boundary value problems:

$$
\left\{\begin{array}{l}
u^{\prime \prime \prime}(t)+a(t) f(u(t))=0, \quad 0<t<1, \\
u(0)=u^{\prime}(0)=0, \\
u^{\prime}(1)-\alpha u^{\prime}(\eta)=\lambda .
\end{array}\right.
$$

The author obtained the existence and nonexistence of positive solutions by applying the Guo-Krasnosel'skii fixed point theorem and Schauder's fixed point theorem.

In [10], Zhang and Wang studied the following nonlinear singular fourth-order boundary value problem:

$$
\left\{\begin{array}{l}
u^{(4)}(t)=f(t, u(t)), \quad 0<t<1 \\
u(0)=u(1)=u^{\prime}(0)=u^{\prime}(1)=0
\end{array}\right.
$$

where the nonlinear term $f(t, u)$ may be singular at $t=0, t=1$ and $u=0$. The author presented the existence of a positive solution by using the fixed point index theorem and the properties of Green's function.

In [15], by applying the Krasnosel'skii fixed point theorem, Graef, Qian and Yang established the existence and nonexistence of positive solutions for the following fourth-order three-point boundary value problem:

$$
\left\{\begin{array}{l}
u^{(4)}(t)=\lambda g(t) f(u(t)), \quad 0<t<1 \\
u(0)=u^{\prime}(1)=u^{\prime \prime}(0)=u^{\prime \prime}(p)-u^{\prime \prime}(1)=0
\end{array}\right.
$$

where $p \in(0,1)$ is a constant.

Inspired and motivated by the works mentioned above, we deal with the existence and nonexistence of positive solutions to problem (1.1) by making use of the fixed point theorem together with the properties of Green's function. The main features of the paper are as follows. Firstly, we apply the Taylor expansion formula to prove a lemma, and then we give a comparison lemma and construct a special cone. Secondly, we present the existence of positive solutions for problem (1.1). To our best knowledge, no paper has considered problem (1.1). The arguments are based upon the fixed point theorem for the special cone.

The paper is organized as follows. In Section 2, we give some properties of Green's function associated with problem (1.1) and construct a suitable cone and transform problem (1.1) into an integral equation. In Section 3, we discuss the existence of at least one positive solution for problem (1.1). 


\section{Preliminary lemmas}

Let $E=C[0,1]$ be a Banach space of all continuous functions with the norm $\|u\|=$ $\max _{0 \leq t \leq 1}|u(t)|, C^{+}[0,1]=\{u \in C[0,1]: u(t)>0, t \in[0,1]\}$.

Throughout the paper, we assume that

$\left(\mathrm{H}_{1}\right) f:(0,1) \times[0,+\infty) \rightarrow[0,+\infty)$ is continuous.

$\left(\mathrm{H}_{2}\right)$ There exists a continuous function $q:(0,1) \rightarrow[0,+\infty)$ such that

$$
0<\int_{a}^{b} s(1-s) q(s) d s \leq \int_{0}^{1} s(1-s) q(s) d s<+\infty \quad \text { for }[a, b] \subset(0,1)
$$

$\left(\mathrm{H}_{3}\right)$ There exists a continuous function $g:[0,1] \times[0,+\infty) \rightarrow[0,+\infty)$ such that

$$
f(t, u) \leq q(t) g(t, u), \quad(t, u) \in(0,1) \times[0,+\infty) .
$$

Lemma 2.1 Suppose that $p(t) \in L^{1}(0,1)$ and $p(t)>0$. Then the linear boundary value problem

$$
\left\{\begin{array}{l}
u^{(4)}(t)+p(t)=0, \\
u(0)=u^{\prime}(0)=u^{\prime \prime}(0)=0, \\
u^{\prime \prime}(1)-\alpha u^{\prime \prime}(\eta)=\lambda
\end{array}\right.
$$

has a unique positive solution, which can be expressed by

$$
u(t)=\int_{0}^{1} G(t, s) p(s) d s+\frac{\alpha t^{3}}{6(1-\alpha \eta)} \int_{0}^{1} K(\eta, s) p(s) d s+\frac{\lambda t^{3}}{6(1-\alpha \eta)},
$$

where

$$
G(t, s)= \begin{cases}\frac{1}{6} t^{3}(1-s)-\frac{1}{6}(t-s)^{3}, & 0 \leq s \leq t \leq 1 \\ \frac{1}{6} t^{3}(1-s), & 0 \leq t \leq s \leq 1\end{cases}
$$

and

$$
\frac{\partial}{\partial t} G(t, s)= \begin{cases}\frac{1}{2} t^{2}(1-s)-\frac{1}{2}(t-s)^{2}, & 0 \leq s \leq t \leq 1, \\ \frac{1}{2} t^{2}(1-s), & 0 \leq t \leq s \leq 1,\end{cases}
$$

and

$$
K(t, s)=\frac{\partial^{2}}{\partial t^{2}} G(t, s)= \begin{cases}s(1-t), & 0 \leq s \leq t \leq 1, \\ t(1-s), & 0 \leq t \leq s \leq 1 .\end{cases}
$$

Proof In fact, if $u(t)$ is a solution of problem (2.1), by the Taylor expansion formula, we have

$$
u(t)=a_{0}+a_{1} t+\frac{a_{2}}{2 !} t^{2}+\frac{a_{3}}{3 !} t^{3}-\frac{1}{6} \int_{0}^{t}(t-s)^{3} p(s) d s
$$


then

$$
\begin{aligned}
& u^{\prime}(t)=a_{1}+a_{2} t+\frac{a_{3}}{2} t^{2}-\frac{1}{2} \int_{0}^{t}(t-s)^{2} p(s) d s, \\
& u^{\prime \prime}(t)=a_{2}+a_{3} t-\int_{0}^{t}(t-s) p(s) d s,
\end{aligned}
$$

which together with the boundary condition implies $a_{0}=a_{1}=a_{2}=0$ and

$$
a_{3}=\frac{1}{1-\alpha \eta} \int_{0}^{1}(1-s) p(s) d s+\frac{\alpha}{1-\alpha \eta} \int_{0}^{\eta}(s-\eta) p(s) d s+\frac{\lambda}{1-\alpha \eta} .
$$

Therefore

$$
\begin{aligned}
u(t)= & -\frac{1}{6} \int_{0}^{t}(t-s)^{3} p(s) d s+\frac{t^{3}}{6(1-\alpha \eta)} \int_{0}^{1}(1-s) p(s) d s \\
& +\frac{\alpha t^{3}}{6(1-\alpha \eta)} \int_{0}^{\eta}(s-\eta) p(s) d s+\frac{\lambda t^{3}}{6(1-\alpha \eta)} \\
= & \frac{1}{6} \int_{0}^{t}\left[t^{3}(1-s)-(t-s)^{3}\right] p(s) d s+\frac{1}{6} \int_{t}^{1} t^{3}(1-s) p(s) d s \\
& +\frac{\alpha t^{3}}{6(1-\alpha \eta)}\left[\int_{0}^{\eta} s(1-\eta) p(s) d s+\int_{\eta}^{1} \eta(1-s) p(s) d s\right]+\frac{\lambda t^{3}}{6(1-\alpha \eta)} \\
= & \int_{0}^{1} G(t, s) p(s) d s+\frac{\alpha t^{3}}{6(1-\alpha \eta)} \int_{0}^{1} K(\eta, s) p(s) d s+\frac{\lambda t^{3}}{6(1-\alpha \eta)} .
\end{aligned}
$$

The proof is complete.

Lemma 2.2 For all $(t, s) \in[0,1] \times[0,1]$, we have

$$
\frac{1}{6} t^{3} s(1-s) \leq G(t, s) \leq s(1-s)
$$

Proof If $0 \leq t \leq s \leq 1$, then

$$
G(t, s)=\frac{1}{6} t^{3}(1-s) \leq \frac{1}{6} s^{3}(1-s) \leq s(1-s)
$$

and

$$
G(t, s)=\frac{1}{6} t^{3}(1-s) \geq \frac{1}{6} t^{3} s(1-s) .
$$

If $0 \leq s \leq t \leq 1$, then

$$
\begin{aligned}
G(t, s) & =\frac{1}{6} t^{3}(1-s)-\frac{1}{6}(t-s)^{3} \\
& \leq \frac{1}{6} s\left[t^{2}-t^{3}+3 t(t-s)\right] \\
& \leq \frac{1}{6} s\left[t^{2}(1-s)+3 t(1-s)\right] \leq s(1-s),
\end{aligned}
$$


and

$$
\begin{aligned}
G(t, s) & =\frac{1}{6} t^{3}(1-s)-\frac{1}{6}(t-s)^{3} \\
& \geq \frac{1}{6} t^{3} s(1-s)+\frac{1}{6} t^{3}(1-s)^{3}-\frac{1}{6}(t-s)^{3} \\
& \geq \frac{1}{6} t^{3} s(1-s)+\frac{1}{6} s(1-t)\left[t^{2}(1-s)^{2}+t(1-s)(t-s)+(t-s)^{2}\right] \\
& \geq \frac{1}{6} t^{3} s(1-s) .
\end{aligned}
$$

Therefore

$$
\frac{1}{6} t^{3} s(1-s) \leq G(t, s) \leq s(1-s)
$$

Define a cone $K \subset C[0,1]$ by

$$
K=\left\{u(t) \in C^{+}[0,1]: u(t) \geq \frac{1}{6} t^{3}\|u\|, 0 \leq t \leq 1\right\},
$$

then $K$ is a positive cone in $C[0,1]$. Denote

$$
\Omega_{r}=\{u \in K:\|u\|<r\}, \quad \partial \Omega_{r}=\{u \in K:\|u\|=r\} .
$$

Fix $R>r>0$. Define an operator $A:\left(\bar{\Omega}_{R} \backslash \Omega_{r}\right) \cap K \rightarrow K$ by

$$
A u(t)=\int_{0}^{1} G(t, s) f(s, u(s)) d s+\frac{\alpha t^{3}}{6(1-\alpha \eta)} \int_{0}^{1} K(\eta, s) f(s, u(s)) d s+\frac{\lambda t^{3}}{6(1-\alpha \eta)} .
$$

It is well known that problem (1.1) has a positive solution $u=u(t)$ if and only if $u$ is a fixed point of $A$.

Lemma 2.3 Suppose that $\left(\mathrm{H}_{1}\right) \sim\left(\mathrm{H}_{3}\right)$ hold. Then $A(K) \subseteq K$.

Proof From $\left(\mathrm{H}_{2}\right)$ and $\left(\mathrm{H}_{3}\right)$, we know that

$$
\begin{aligned}
0 & \leq(A u)(t) \\
& \leq \int_{0}^{1} s(1-s) f(s, u(s)) d s+\frac{\alpha}{6(1-\alpha \eta)} \int_{0}^{1} K(\eta, s) f(s, u(s)) d s+\frac{\lambda}{6(1-\alpha \eta)} \\
& \leq \int_{0}^{1} s(1-s) q(s) g(s, u(s)) d s+\frac{\alpha}{6(1-\alpha \eta)} \int_{0}^{1} K(\eta, s) q(s) g(s, u(s)) d s+\frac{\lambda}{6(1-\alpha \eta)} \\
& <+\infty
\end{aligned}
$$

On the other hand, for any $u \in K$, we have $u(t) \geq \frac{1}{6} t^{3}\|u\|, t \in[0,1]$, and

$$
\|A u\| \leq \int_{0}^{1} s(1-s) f(s, u(s)) d s+\frac{\alpha}{1-\alpha \eta} \int_{0}^{1} K(\eta, s) f(s, u(s)) d s+\frac{\lambda}{1-\alpha \eta} .
$$


Therefore

$$
\begin{aligned}
(A u)(t) & \geq \frac{1}{6} t^{3}\left[\int_{0}^{1} s(1-s) f(s, u(s)) d s+\frac{\alpha}{1-\alpha \eta} \int_{0}^{1} K(\eta, s) f(s, u(s)) d s+\frac{\lambda}{1-\alpha \eta}\right] \\
& \geq \frac{1}{6} t^{3}\|A u\| .
\end{aligned}
$$

The proof is complete.

Lemma 2.4 Suppose that $\left(\mathrm{H}_{1}\right) \sim\left(\mathrm{H}_{3}\right)$ hold. Then $A:\left(\bar{\Omega}_{R} \backslash \Omega_{r}\right) \cap K \rightarrow K$ is completely continuous.

Proof For any $u \in\left(\bar{\Omega}_{R} \backslash \Omega_{r}\right) \cap K$, we have $0 \leq \frac{1}{6} t^{3} r \leq \frac{1}{6} t^{3}\|u\| \leq u(t) \leq R$.

Let

$$
q_{n}(t)= \begin{cases}\inf _{t \leq s \leq \frac{1}{n}} q(s), & 0 \leq t \leq \frac{1}{n} \\ q(t), & \frac{1}{n} \leq t \leq \frac{n-1}{n} \\ \inf _{\frac{n-1}{n} \leq s \leq t} q(s), & \frac{n-1}{n} \leq t \leq 1\end{cases}
$$

Then, from $\left(\mathrm{H}_{2}\right)$ and $\left(\mathrm{H}_{3}\right)$, we have $\lim _{n \rightarrow \infty} \int_{0}^{1}\left(q(t)-q_{n}(t)\right) d t=0$ for $0 \leq t \leq 1$ and $u \in K$. Let

$$
f_{n}(t, u)= \begin{cases}f(t, u), & f(t, u) \leq q_{n}(t) g(t, u), \\ q_{n}(t) g(t, u), & f(t, u)>q_{n}(t) g(t, u) .\end{cases}
$$

It is easy to see that $f_{n}$ is a continuous function on $[0,1] \times[0,+\infty)$ and $f_{n}$ is bounded on any bounded set. Define

$$
\left(A_{n} u\right)(t)=\int_{0}^{1} G(t, s) f_{n}(s, u(s)) d s+\frac{\alpha t^{3}}{6(1-\alpha \eta)} \int_{0}^{1} K(\eta, s) f_{n}(s, u(s)) d s+\frac{\lambda t^{3}}{6(1-\alpha \eta)} .
$$

By the Arzela-Ascoli theorem, we know that $A_{n}: \bar{\Omega}_{R} \backslash \Omega_{r} \rightarrow C[0,1]$ is completely continuous.

Let $M(R)=\max \{g(t, u):(t, u) \in[0,1] \times[0, R]\}$. For $u \in\left(\bar{\Omega}_{R} \backslash \Omega_{r}\right) \cap K$, we know that

$$
\begin{aligned}
\left\|A u-A_{n} u\right\|= & \max _{0 \leq t \leq 1}\left\{\int_{0}^{1} G(t, s)\left[f(s, u(s))-f_{n}(s, u(s))\right] d s\right\} \\
& +\max _{0 \leq t \leq 1}\left\{\frac{\alpha t^{3}}{6(1-\alpha \eta)} \int_{0}^{1} K(\eta, s)\left[f(s, u(s))-f_{n}(s, u(s))\right] d s\right\} \\
\leq & \max _{0 \leq t \leq 1}\left\{\int_{0}^{1} G(t, s)\left[q(s) g(s, u(s))-q_{n}(s) g(s, u(s))\right] d s\right\} \\
& +\max _{0 \leq t \leq 1}\left\{\frac{\alpha t^{3}}{6(1-\alpha \eta)} \int_{0}^{1} K(\eta, s)\left[q(s) g(s, u(s))-q_{n}(s) g(s, u(s))\right] d s\right\} \\
\leq & M(R) \max _{0 \leq t \leq 1}\left\{\int_{0}^{1} G(t, s)\left[q(s)-q_{n}(s)\right] d s\right\}
\end{aligned}
$$




$$
\begin{aligned}
& \quad+M(R) \max _{0 \leq t \leq 1}\left\{\frac{\alpha t^{3}}{6(1-\alpha \eta)} \int_{0}^{1} K(\eta, s)\left[q(s)-q_{n}(s)\right] d s\right\} \\
& \rightarrow 0 \quad \text { as } n \rightarrow \infty .
\end{aligned}
$$

It shows that a completely continuous operator $A_{n}$ converges to an operator $A$ uniformly on $\left(\bar{\Omega}_{R} \backslash \Omega_{r}\right) \cap K$. Hence $A$ is continuous.

Suppose that $D \subset K$ is a bounded set, then there exists $d>0$ such that $\|u\| \leq d$ for any $u \in D$. From $\left(\mathrm{H}_{3}\right)$, we know that $|f(t, u)| \leq q(t) g(t, u) \leq M(d) q(t)$ for $(t, u) \in(0,1) \times[0, d]$. Then we have

$$
\begin{aligned}
\|A u\| & \leq \int_{0}^{1} s(1-s) f(s, u(s)) d s+\frac{\alpha}{6(1-\alpha \eta)} \int_{0}^{1} K(\eta, s) f(s, u(s)) d s+\frac{\lambda}{6(1-\alpha \eta)} \\
& \leq \int_{0}^{1} s(1-s) q(s) g(s, u(s)) d s+\frac{\alpha}{6(1-\alpha \eta)} \int_{0}^{1} K(\eta, s) q(s) g(s, u(s)) d s+\frac{\lambda}{6(1-\alpha \eta)} \\
& \leq M(d) \int_{0}^{1} s(1-s) q(s) d s+M(d) \frac{\alpha}{6(1-\alpha \eta)} \int_{0}^{1} K(\eta, s) q(s) d s+\frac{\lambda}{6(1-\alpha \eta)} \\
& <+\infty .
\end{aligned}
$$

Hence $A$ is uniformly bounded.

On the other hand, for any $u \in D$, we know that

$$
\begin{aligned}
\left|(A u)^{\prime}(t)\right| \leq & \frac{1}{2} \int_{0}^{t} s(1-s) f(s, u(s)) d s+\frac{1}{2} \int_{t}^{1} s(1-s) f(s, u(s)) d s \\
& +\frac{\alpha t^{2}}{2(1-\alpha \eta)} \int_{0}^{1} K(\eta, s) f(s, u(s)) d s+\frac{\lambda t^{2}}{2(1-\alpha \eta)} \\
\leq & \frac{1}{2} \int_{0}^{t} s(1-s) q(s) g(s, u(s)) d s+\frac{1}{2} \int_{t}^{1} s(1-s) q(s) g(s, u(s)) d s \\
& +\frac{\alpha t^{2}}{2(1-\alpha \eta)} \int_{0}^{1} K(\eta, s) q(s) g(s, u(s)) d s+\frac{\lambda t^{2}}{2(1-\alpha \eta)} \\
\leq & \frac{1}{2} M(d) \int_{0}^{t} s(1-s) q(s) d s+\frac{1}{2} M(d) \int_{t}^{1} s(1-s) q(s) d s \\
& +\frac{\alpha t^{2}}{2(1-\alpha \eta)} M(d) \int_{0}^{1} K(\eta, s) q(s) d s+\frac{\lambda t^{2}}{2(1-\alpha \eta)} .
\end{aligned}
$$

Let

$$
\begin{aligned}
\varphi(t)=\frac{1}{2} M(d) & \int_{0}^{1} s(1-s) q(s) d s+\frac{\alpha t^{2}}{2(1-\alpha \eta)} M(d) \int_{0}^{1} K(\eta, s) q(s) d s+\frac{\lambda t^{2}}{2(1-\alpha \eta)} \\
\int_{0}^{1}|\varphi(t)| d t= & M(d) \int_{0}^{1} d t \int_{0}^{t} \frac{1}{2} s(1-s) q(s) d s+M(d) \int_{0}^{1} d t \int_{t}^{1} \frac{1}{2} s(1-s) q(s) d s \\
& +M(d) \int_{0}^{1} \frac{\alpha t^{2}}{2(1-\alpha \eta)} d t \int_{0}^{1} K(\eta, s) q(s) d s+\int_{0}^{1} \frac{\lambda t^{2}}{2(1-\alpha \eta)} d t \\
= & M(d) \int_{0}^{1} \frac{1}{2} s(1-s)^{2} q(s) d s+M(d) \int_{0}^{1} \frac{1}{2} s^{2}(1-s) q(s) d s \\
& +\frac{\alpha}{6(1-\alpha \eta)} M(d) \int_{0}^{1} K(\eta, s) q(s) d s+\frac{\lambda}{6(1-\alpha \eta)}
\end{aligned}
$$




$$
\begin{aligned}
& =\frac{1}{2} M(d) \int_{0}^{1} s(1-s) q(s) d s+\frac{\alpha}{6(1-\alpha \eta)} M(d) \int_{0}^{1} K(\eta, s) q(s) d s+\frac{\lambda}{6(1-\alpha \eta)} \\
& <+\infty
\end{aligned}
$$

Therefore $A$ is equicontinuous. Consequently, $A$ is completely continuous.

Lemma $2.5[21,22]$ Let $E$ be a Banach space, and let $P \subset E$ be a cone in $E$. Assume that $\Omega_{1}$ and $\Omega_{2}$ are open subsets of $E$ with $0 \in \Omega_{1}$ and $\bar{\Omega}_{1} \subset \Omega_{2}$. Let $T: P \cap\left(\bar{\Omega}_{2} \backslash \Omega_{1}\right) \rightarrow P$ be a completely continuous operator such that either

(i) $\|T u\| \leq\|u\|, u \in P \cap \partial \Omega_{1}$ and $\|T u\| \geq\|u\|, u \in P \cap \partial \Omega_{2}$, or

(ii) $\|T u\| \geq\|u\|, u \in P \cap \partial \Omega_{1}$ and $\|T u\| \leq\|u\|, u \in P \cap \partial \Omega_{2}$.

Then $T$ has a fixed point in $P \cap\left(\bar{\Omega}_{2} \backslash \Omega_{1}\right)$.

\section{Main results}

Theorem 3.1 Suppose that $\left(\mathrm{H}_{1}\right),\left(\mathrm{H}_{2}\right)$ and $\left(\mathrm{H}_{3}\right)$ hold. In addition, assume that the following conditions hold:

$\left(\mathrm{H}_{4}\right) \lim _{u \rightarrow 0^{+}} \sup \max _{0 \leq t \leq 1} \frac{g(t, u)}{u}=0$;

$\left(\mathrm{H}_{5}\right) \lim _{u \rightarrow+\infty} \inf \min _{a \leq t \leq b} \frac{f(t, u)}{u}=+\infty$.

Then problem (1.1) has at least one positive solution for $\lambda$ small enough, and problem (1.1) has no positive solution for $\lambda$ large enough.

Proof For $\lambda>0$ small enough, let

$$
N=\frac{5}{6}\left[\int_{0}^{1} s(1-s) q(s) d s+\frac{\alpha}{6(1-\alpha \eta)} \int_{0}^{1} K(\eta, s) q(s) d s\right]^{-1} .
$$

From $\left(\mathrm{H}_{4}\right)$, there exists a constant $R_{1}>0$ such that $g(t, u) \leq N u$ for $(t, u) \in[0,1] \times\left(0, R_{1}\right]$.

Let $\Omega_{1}=\left\{u \in K:\|u\|<R_{1}\right\}, 0<\lambda \leq(1-\alpha \eta) R_{1}$. For any $u \in K \cap \partial \Omega_{1}$, we get

$$
\begin{aligned}
A u(t) & =\int_{0}^{1} G(t, s) f(s, u(s)) d s+\frac{\alpha t^{3}}{6(1-\alpha \eta)} \int_{0}^{1} K(\eta, s) f(s, u(s)) d s+\frac{\lambda t^{3}}{6(1-\alpha \eta)} \\
& \leq \int_{0}^{1} s(1-s) f(s, u(s)) d s+\frac{\alpha}{6(1-\alpha \eta)} \int_{0}^{1} K(\eta, s) f(s, u(s)) d s+\frac{\lambda}{6(1-\alpha \eta)} \\
& \leq \int_{0}^{1} s(1-s) q(s) g(s, u(s)) d s+\frac{\alpha}{6(1-\alpha \eta)} \int_{0}^{1} K(\eta, s) q(s) g(s, u(s)) d s+\frac{\lambda}{6(1-\alpha \eta)} \\
& \leq N \int_{0}^{1} s(1-s) q(s) u(s) d s+\frac{N \alpha}{6(1-\alpha \eta)} \int_{0}^{1} K(\eta, s) q(s) u(s) d s+\frac{R_{1}(1-\alpha \eta)}{6(1-\alpha \eta)} \\
& \leq N\|u\| \int_{0}^{1} s(1-s) q(s) d s+\frac{N \alpha\|u\|}{6(1-\alpha \eta)} \int_{0}^{1} K(\eta, s) q(s) d s+\frac{R_{1}}{6} \\
& =\frac{5}{6} R_{1}+\frac{1}{6} R_{1}=R_{1}=\|u\| .
\end{aligned}
$$

Therefore

$$
\|A u\| \leq\|u\|, \quad u \in K \cap \partial \Omega_{1} .
$$


On the other hand, let

$$
M=\left[\int_{a}^{b} \frac{1}{6} a^{3} s(1-s) d s+\frac{\alpha a^{3}}{6(1-\alpha \eta)} \int_{a}^{b} K(\eta, s) d s\right]^{-1} .
$$

From $\left(\mathrm{H}_{5}\right)$, there exists $R>0$ such that $f(t, u) \geq 48 M u$ for $(t, u) \in[a, b] \times[R,+\infty)$. Let $R_{2}>\frac{6 R}{a^{3}}>R_{1}$, and let $\Omega_{2}=\left\{u \in K:\|u\|<R_{2}\right\}$. For any $u \in K \cap \partial \Omega_{2}$ and $t \in[a, b]$, we know that $u(t) \geq \frac{1}{6} t^{3}\|u\|=\frac{1}{6} a^{3} R_{2}>R$ and

$$
\begin{aligned}
(A u)\left(\frac{1}{2}\right)= & \int_{0}^{1} G\left(\frac{1}{2}, s\right) f(s, u(s)) d s \\
& +\frac{1}{8} \cdot \frac{\alpha}{6(1-\alpha \eta)} \int_{0}^{1} K(\eta, s) f(s, u(s)) d s+\frac{1}{8} \cdot \frac{\lambda}{6(1-\alpha \eta)} \\
\geq & \frac{1}{8} \int_{0}^{1} \frac{1}{6} s(1-s) f(s, u(s)) d s+\frac{\alpha}{48(1-\alpha \eta)} \int_{0}^{1} K(\eta, s) f(s, u(s)) d s \\
\geq & \frac{1}{8} \int_{a}^{b} \frac{1}{6} s(1-s) f(s, u(s)) d s+\frac{\alpha}{48(1-\alpha \eta)} \int_{a}^{b} K(\eta, s) f(s, u(s)) d s \\
\geq & M \int_{a}^{b} s(1-s) u(s) d s+\frac{\alpha M}{1-\alpha \eta} \int_{a}^{b} K(\eta, s) u(s) d s \\
\geq & M \int_{a}^{b} \frac{1}{6} a^{3} s(1-s) u(s) d s+\frac{\alpha M a^{3}}{6(1-\alpha \eta)} \int_{a}^{b} K(\eta, s) u(s) d s \\
= & M\left[\int_{a}^{b} \frac{1}{6} a^{3} s(1-s) d s+\frac{\alpha a^{3}}{6(1-\alpha \eta)} \int_{a}^{b} K(\eta, s) d s\right]\|u\| \\
= & \|u\|,
\end{aligned}
$$

which implies that

$$
\|A u\| \geq\|u\| \quad \text { for } u \in K \cap \partial \Omega_{2} .
$$

By Lemma 2.5 we know that problem (1.1) has at least one positive solution.

For $\lambda$ large enough, we prove that problem (1.1) has no positive solution. Otherwise, there exists $0<\lambda_{1}<\lambda_{2}<\lambda_{3}<\cdots<\lambda_{n}<\cdots$ with $\lim _{n \rightarrow \infty} \lambda_{n}=+\infty$ such that problem (1.1) has a positive solution $u_{n}(t)$, then we get

$$
\begin{aligned}
u_{n}\left(\frac{1}{2}\right)= & \int_{0}^{1} G\left(\frac{1}{2}, s\right) f\left(s, u_{n}(s)\right) d s \\
& +\frac{\alpha}{48(1-\alpha \eta)} \int_{0}^{1} K(\eta, s) f\left(s, u_{n}(s)\right) d s+\frac{\lambda_{n}}{48(1-\alpha \eta)} \\
\geq & \frac{\lambda_{n}}{48(1-\alpha \eta)} \rightarrow+\infty \quad \text { as } n \rightarrow+\infty .
\end{aligned}
$$

Hence $\left\|u_{n}\right\| \rightarrow+\infty$ as $n \rightarrow+\infty$.

Again from $\left(\mathrm{H}_{5}\right)$, there exist $R^{\prime}>0$ and $M>0$ such that

$$
f(t, u) \geq 96 M u \quad \text { for }(t, u) \in[a, b] \times\left[R^{\prime},+\infty\right)
$$


where $M$ is defined by (3.2). Let $n$ be large enough. Choose $R_{2}^{\prime}>\frac{6 a^{3}}{R^{\prime}}$ such that $\left\|u_{n}\right\| \geq R_{2}^{\prime}$. Thus, we get

$$
\begin{aligned}
\left\|u_{n}\right\| \geq & u_{n}\left(\frac{1}{2}\right) \\
= & \int_{0}^{1} G\left(\frac{1}{2}, s\right) f\left(s, u_{n}(s)\right) d s \\
& +\frac{\alpha}{48(1-\alpha \eta)} \int_{0}^{1} K(\eta, s) f\left(s, u_{n}(s)\right) d s+\frac{\lambda_{n}}{48(1-\alpha \eta)} \\
\geq & \frac{1}{8} \int_{0}^{1} \frac{1}{6} s(1-s) f\left(s, u_{n}(s)\right) d s+\frac{\alpha}{48(1-\alpha \eta)} \int_{0}^{1} K(\eta, s) f\left(s, u_{n}(s)\right) d s \\
\geq & \frac{1}{8} \int_{a}^{b} \frac{1}{6} s(1-s) f\left(s, u_{n}(s)\right) d s+\frac{\alpha}{48(1-\alpha \eta)} \int_{a}^{b} K(\eta, s) f\left(s, u_{n}(s)\right) d s \\
\geq & 2 M \int_{a}^{b} s(1-s) u_{n}(s) d s+\frac{2 M \alpha}{1-\alpha \eta} \int_{a}^{b} K(\eta, s) u_{n}(s) d s \\
\geq & 2 M\left[\int_{a}^{b} \frac{1}{6} a^{3} s(1-s) d s+\frac{\alpha a^{3}}{6(1-\alpha \eta)} \int_{a}^{b} K(\eta, s) d s\right]\left\|u_{n}\right\|=2\left\|u_{n}\right\|,
\end{aligned}
$$

which is a contradiction. The proof is complete.

Remark 3.1 The conclusion of Theorem 3.1 also holds if $\lambda=0$.

Theorem 3.2 Suppose that $\left(\mathrm{H}_{1}\right),\left(\mathrm{H}_{2}\right)$ and $\left(\mathrm{H}_{3}\right)$ hold. In addition, assume that

$\left(\mathrm{H}_{6}\right) \lim _{u \rightarrow+\infty} \inf \min _{0 \leq t \leq 1} \frac{g(t, u)}{u}=0$;

$\left(\mathrm{H}_{7}\right) \lim _{u \rightarrow 0^{+}} \sup \max _{a \leq t \leq b} \frac{f(t, u)}{u}=+\infty$.

Then problem (1.1) has at least one positive solution for any $\lambda \in[0,+\infty)$.

Proof From $\left(\mathrm{H}_{7}\right)$, there exist constants $R_{1}>0$ and $M>0$ such that

$$
f(t, u) \geq 48 M u \quad \text { for }(t, u) \in[a, b] \times\left(0, R_{1}\right]
$$

where $M$ is defined by (3.2). Let $\Omega_{1}=\left\{u \in K:\|u\|<R_{1}\right\}$. For any $u \in K \cap \partial \Omega_{1}$, we know that

$$
\begin{aligned}
(A u)\left(\frac{1}{2}\right)= & \int_{0}^{1} G\left(\frac{1}{2}, s\right) f(s, u(s)) d s \\
& +\frac{\alpha}{48(1-\alpha \eta)} \int_{0}^{1} K(\eta, s) f(s, u(s)) d s+\frac{\lambda}{48(1-\alpha \eta)} \\
\geq & \frac{1}{8} \int_{0}^{1} \frac{1}{6} s(1-s) f(s, u(s)) d s+\frac{\alpha}{48(1-\alpha \eta)} \int_{0}^{1} K(\eta, s) f(s, u(s)) d s \\
\geq & \frac{1}{8} \int_{a}^{b} \frac{1}{6} s(1-s) f(s, u(s)) d s+\frac{\alpha}{48(1-\alpha \eta)} \int_{a}^{b} K(\eta, s) f(s, u(s)) d s \\
\geq & M \int_{a}^{b} s(1-s) u(s) d s+\frac{M \alpha}{1-\alpha \eta} \int_{a}^{b} K(\eta, s) u(s) d s \\
\geq & M\left[\int_{a}^{b} \frac{1}{6} a^{3} s(1-s) d s+\frac{\alpha a^{3}}{6(1-\alpha \eta)} \int_{a}^{b} K(\eta, s) d s\right]\|u\|=\|u\| .
\end{aligned}
$$


Therefore

$\|A u\| \geq\|u\| \quad$ for $u \in K \cap \partial \Omega_{1}$.

On the other hand, from $\left(\mathrm{H}_{6}\right)$, there exists a constant $R_{0}>0$ such that $g(t, u) \leq N u$ for $u \geq R_{0}$, where $N$ is defined by (3.1). Since $g(t, u)$ is continuous on $[0,1] \times[0,+\infty)$, there exists $M^{*}>0$ such that $\max _{0 \leq t \leq 1} g(t, u) \leq M^{*}$ for $(t, u) \in[0,1] \times\left[0, R_{0}\right]$. Choose

$$
R_{2} \geq \max \left\{2 R_{1}, \frac{M^{*}}{N}, R_{0}, \frac{\lambda}{1-\alpha \eta}\right\}
$$

Let $\Omega_{2}=\left\{u \in K:\|u\|<R_{2}\right\}$. For any $u \in\left[0, R_{2}\right]$, we get

$$
f(t, u) \leq q(t) g(t, u) \leq \frac{1}{2} M^{*}+\frac{1}{2} N R_{2} .
$$

Thus, for any $u \in K \cap \partial \Omega_{2}$ and $t \in(0,1)$, we know that

$$
\begin{aligned}
A u(t)= & \int_{0}^{1} G(t, s) f(s, u(s)) d s+\frac{\alpha t^{3}}{6(1-\alpha \eta)} \int_{0}^{1} K(\eta, s) f(s, u(s)) d s+\frac{\lambda t^{3}}{6(1-\alpha \eta)} \\
\leq & \int_{0}^{1} s(1-s) f(s, u(s)) d s+\frac{\alpha}{6(1-\alpha \eta)} \int_{0}^{1} K(\eta, s) f(s, u(s)) d s+\frac{\lambda}{6(1-\alpha \eta)} \\
\leq & \int_{0}^{1} s(1-s) q(s) g(s, u(s)) d s+\frac{\alpha}{6(1-\alpha \eta)} \int_{0}^{1} K(\eta, s) q(s) g(s, u(s)) d s+\frac{\lambda}{6(1-\alpha \eta)} \\
\leq & \int_{0}^{1} s(1-s) q(s)\left(\frac{1}{2} M^{*}+\frac{1}{2} N R_{2}\right) d s \\
& +\frac{\alpha}{6(1-\alpha \eta)} \int_{0}^{1} K(\eta, s) q(s)\left(\frac{1}{2} M^{*}+\frac{1}{2} N R_{2}\right) d s+\frac{1}{6} R_{2} \\
\leq & \frac{1}{2} M^{*}\left[\int_{0}^{1} s(1-s) q(s) d s+\frac{\alpha}{6(1-\alpha \eta)} \int_{0}^{1} K(\eta, s) q(s) d s\right] \\
& +\frac{1}{2} N R_{2}\left[\int_{0}^{1} s(1-s) q(s) d s+\frac{\alpha}{6(1-\alpha \eta)} \int_{0}^{1} K(\eta, s) q(s) d s\right]+\frac{1}{6} R_{2} \\
\leq & \frac{5 M^{*}}{12 N}+\frac{5}{12 N} N R_{2}+\frac{1}{6} R_{2} \\
\leq & \frac{5}{12} R_{2}+\frac{5}{12} R_{2}+\frac{1}{6} R_{2}=R_{2}=\|u\| .
\end{aligned}
$$

Therefore

$\|A u\| \leq\|u\| \quad$ for $u \in K \cap \partial \Omega_{2}$.

It follows from Lemma 2.5 that problem (1.1) has at least one positive solution.

\section{Examples}

Now, we give examples to illustrate the main results in the paper. 
Example 4.1 Consider the following boundary value problem:

$$
\left\{\begin{array}{l}
u^{(4)}(t)+\frac{1}{t^{1+\omega_{1}+\omega_{2}+\omega_{3}(1-t)^{3+\omega_{4}}}}\left(u^{2}+\sqrt{u}\right) \sin ^{2} 2 u=0, \quad 0<t<1, \\
u(0)=u^{\prime}(0)=u^{\prime \prime}(0)=0, \\
u^{\prime \prime}(1)-\frac{1}{3} u^{\prime \prime}\left(\frac{1}{2}\right)=1 .
\end{array}\right.
$$

Then problem (4.1) has at least one positive solution if $\omega_{1}+\omega_{2}+\omega_{3}<1$ and $\omega_{4}<-1$.

Let

$$
q(t)=\frac{1}{t^{1+\omega_{1}+\omega_{2}+\omega_{3}}(1-t)^{3+\omega_{4}}}, \quad g(t, u)=2\left(u^{2}+\sqrt{u}\right) \sin ^{2} 2 u .
$$

Take $[a, b]=\left[\frac{1}{4}, \frac{3}{4}\right]$. Notice, for any fixed $t \in(0,1)$, that $f(t, x) \leq q(t) g(t, x)$ and $0<\int_{0}^{1} s(1-$ s) $q(s) d s<+\infty$ for $\omega_{1}+\omega_{2}+\omega_{3}<1$ and $\omega_{4}<-1$.

Obviously, conditions $\left(\mathrm{H}_{1}\right) \sim\left(\mathrm{H}_{3}\right)$ are satisfied.

Now, for any fixed $t \in(0,1),\left(\mathrm{H}_{4}\right)$ and $\left(\mathrm{H}_{5}\right)$ follow immediately from

$$
\begin{aligned}
& \lim _{u \rightarrow 0^{+}} \sup \max _{0 \leq t \leq 1} \frac{2\left(u^{2}+\sqrt{u}\right) \sin ^{2} 2 u}{u}=0, \\
& \lim _{u \rightarrow+\infty} \inf \min _{\frac{1}{4} \leq t \leq \frac{3}{4}} \frac{\left(u^{2}+\sqrt{u}\right) \sin ^{2} 2 u}{t^{1+\omega_{1}+\omega_{2}+\omega_{3}}(1-t)^{3+\omega_{4}} u}=+\infty .
\end{aligned}
$$

Thus, the existence of a positive solution follows from Theorem 3.1 if $\omega_{1}+\omega_{2}+\omega_{3}<1$ and $\omega_{4}<-1$.

Example 4.2 Consider the following boundary value problem:

$$
\left\{\begin{array}{l}
u^{(4)}(t)+\frac{2,012|\ln u(t)|+\sin ^{2} u(t)}{\sqrt{t(1-t)}}=0, \quad 0<t<1 \\
u(0)=u^{\prime}(0)=u^{\prime \prime}(0)=0 \\
u^{\prime \prime}(1)-\frac{1}{12} u^{\prime \prime}\left(\frac{1}{6}\right)=2,013
\end{array}\right.
$$

Then problem (4.3) has at least one positive solution.

Let

$$
q(t)=\frac{1}{\sqrt{t(1-t)}}, \quad g(t, u)=2,013|\ln u(t)|+\sin ^{2} u(t) .
$$

Take $[a, b]=\left[\frac{1}{4}, \frac{3}{4}\right]$. Notice, for any fixed $t \in(0,1)$, that $f(t, x) \leq q(t) g(t, x)$ and $0<\int_{0}^{1} s(1-$ s) $q(s) d s<+\infty$.

Obviously, conditions $\left(\mathrm{H}_{1}\right) \sim\left(\mathrm{H}_{3}\right)$ are satisfied.

Now, for any fixed $t \in(0,1),\left(\mathrm{H}_{6}\right)$ and $\left(\mathrm{H}_{7}\right)$ follow immediately from

$$
\begin{aligned}
& \lim _{u \rightarrow 0^{+}} \sup _{\max _{4} \leq t \leq \frac{3}{4}} \frac{2,012|\ln u(t)|+\sin ^{2} u(t)}{\sqrt{t(1-t)} u}=+\infty, \\
& \lim _{u \rightarrow+\infty} \inf \min _{0 \leq t \leq 1} \frac{2,012|\ln u(t)|+\sin ^{2} u(t)}{u}=0 .
\end{aligned}
$$

Thus, the existence of a positive solution follows from Theorem 3.2. 


\section{Competing interests}

The authors declare that they have no competing interests.

\section{Authors' contributions}

The authors declare that the work was realized in collaboration with same responsibility. All authors read and approved the final manuscript.

\section{Author details}

'School of Mathematical Sciences, Fudan University, Shanghai, 200433, P.R. China. ${ }^{2}$ Department of Mathematics, Shanghai Normal University, Shanghai, 200234, P.R. China. ${ }^{3}$ Jiangsu Suzhou Xujiang Experimental Middle School, Suzhou, Jiangsu 215011, P.R. China.

\section{Acknowledgements}

The authors would like to express their thanks to the editor of the journal and the anonymous referees for their careful reading of the first draft of the manuscript and making many helpful comments and suggestions which improved the presentation of the paper. The authors were supported financially by the Foundation of Shanghai Municipal Education Commission (Grant No. DYL201105).

Received: 24 July 2012 Accepted: 20 February 2013 Published: 8 March 2013

\section{References}

1. Ma, R, Zhang, J, Fu, S: The method of upper and lower solutions for fourth-order two point boundary value problems. J. Math. Anal. Appl. 215, 415-422 (1997)

2. Aftabizadeh, AR: Existence and uniqueness theorems for fourth-order boundary value problems. J. Math. Anal. Appl. $116,415-426(1986)$

3. Yang, Y: Fourth-order two-point boundary value problems. Proc. Am. Math. Soc. 104, 175-180 (1988)

4. Gupta, CP: Existence and uniqueness results for the bending of an elastic beam equation at resonance. J. Math. Anal. Appl. 135, 208-225 (1988)

5. Wei, Z: Positive solutions of singular boundary value problems of fourth-order differential equations. Acta Math. Sin 42, 715-722 (1999)

6. Ma, R, Wang, H: On the existence of positive solutions of fourth order ordinary differential equation. Appl. Anal. 59, 225-231 (1995)

7. Harjani, J, López, B, Sadarangani, K: Fixed point theorems for mixed monotone operators and applications to integral equations. Nonlinear Anal. 74, 1749-1760 (2011)

8. Yao, Q: Existence and multiplicity of positive solutions to a class of nonlinear cantilever beam equations. J. Syst. Sci. Math. Sci. 1, 63-69 (2009)

9. Sun, Y: Positive solutions for third-order three-point nonhomogeneous boundary value problems. Appl. Math. Lett. 22, 45-51 (2009)

10. Zhang, K, Wang, C: The existence of positive solutions of a class of fourth-order singular boundary value problems. Acta Math. Sci., Ser. A 29, 127-135 (2009)

11. O'Regan, D: Solvability of some fourth (and higher) order singular boundary value problems. J. Math. Anal. Appl. 161, 78-116 (1991)

12. Schroder, J: Fourth order two-point boundary value problems; estimates by two-sided bounds. Nonlinear Anal. 8 , 107-114 (1984)

13. Guo, L, Sun, J, Zhao, Y: Existence of positive solution for nonlinear third-order three-point boundary value problem. Nonlinear Anal. 68, 3151-3158 (2008)

14. Bai, Z, Wang, H: On the positive solutions of some nonlinear fourth-order beam equations. J. Math. Anal. Appl. 270, 357-368 (2002)

15. Graef, JR, Qian, C, Yang, B: A three point boundary value problem for nonlinear fourth-order differential equations J. Math. Anal. Appl. 287, 217-233 (2003)

16. Yao, Q: Existence and iteration of $n$ symmetric positive solutions for a singular two-point boundary value problem. Comput. Math. Appl. 47, 1195-1200 (2004)

17. Yao, Q: Local existence of multiple positive solutions to a singular cantilever beam equation. J. Math. Anal. Appl. 363, 138-154 (2010)

18. Agarwal, RP: On fourth order boundary value problems arising in beam analysis. Differ. Integral Equ. 2, 91-110 (1989)

19. Ji, C, O'Regan, D, Yan, B, Agarwal, RP: Nonexistence and existence of positive solutions for second order singular three-point boundary value problems with derivative dependent and sign-changing nonlinearities. J. Appl. Math. Comput. 36, 61-87 (2011)

20. Sun, Y, Liu, L, Zhang, J, Agarwal, RP: Positive solutions of singular three-point boundary value problems for second order differential equations. J. Comput. Appl. Math. 230, 738-750 (2009)

21. Guo, D, Lakshmikantham, V: Nonlinear Problems in Abstract Cones. Academic Press, New York (1988)

22. Deimling, K: Nonlinear Functional Analysis. Springer, New York (1985)

doi:10.1186/1687-1847-2013-51

Cite this article as: Sun and Zhu: Existence of positive solutions for singular fourth-order three-point boundary value problems. Advances in Difference Equations 2013 2013:51. 